\title{
Evaluation of the equivocal test results of Treponema pallidum haemagglutination assay
}

\author{
S J Su, S Huang, C Y Chung, H M Yang, Y O Chow
}

Department of
Laboratory Medicine,
Mackay Memorial
Hospital, Taipei,
Taiwan, Republic of
China
S J Su
S S Huang
C Y Chung
H M Yang
Y O Chow
Correspondence to:
Dr S J Su, Serologic Section,
Department of Laboratory
Medicine, Mackay Memorial
Hospital, 92 , Sec. 2, Chung
San North Road, Taipei,
Taiwan 10449 , Republic of
China.
Accepted for publication
12 October 1989

Department of Laboratory Medicine, Taiwan, Republic of China

Y O Chow

Correspondence to: Medicine, Mackay Memoria Hospital, 92, Sec. 2, Chung Taiwan 10449, Republic of

Accepted for publication 12 October 1989

\begin{abstract}
Two hundred and eighty Rapid Plasma Reagin (RPR) positive sera with an emphasis on cases with negative and borderline positive Treponema pallidum haemagglutination assay (TPHA) results were selected. Modified TPHA (MTPHA) and fluorescent treponemal antibody absorption (FTA-abs) tests were used for comparison. One hundred and twenty five samples were TPHA negative, of which 78 and 69 cases were also negative by M-TPHA and FTA-abs, respectively. Eighty one sera negative by TPHA at a titre of $1 / 80$ and positive at $1 / 40$, considered to be negative according to the manufacturer's instructions, were also negative by M-TPHA $(n=11)$ and by FTA-abs $(n=1)$. Fifty borderline positive TPHA specimens gave one negative result by both M-TPHA and FTA-abs. The remaining 24 sera were positive by all three tests.

Because of the high percentage of TPHA negative results among the positive RPR sera which became reactive when rechecked by the FTA-abs, it is concluded that as a confirmatory test the TPHA should be used not instead of but in addition to the FTA-abs.
\end{abstract}

In terms of its prevalence and morbidity syphilis is still an important sexually transmitted disease in Taiwan. Forty thousand four hundred and seventeen sera were sent for routine screening of syphilis during the period from January to November 1988 at this laboratory. Two thousand one hundred $\left(5_{0}^{\circ}\right)$ samples were reactive for Rapid Plasma Reagin (RPR), among which $1126 \quad\left(54^{\circ}{ }_{0}\right)$ were positive for Treponema pallidum haemagglutination assay (TPHA).

RPR is routinely used as a screening test at this laboratory with TPHA, instead of the fluorescent treponemal antibody absorption test (FTA-abs) as a confirmatory test because it is easier to perform and read. Because of the ongoing dilemma about the reporting of equivocal TPHA results, we decided to compare the TPHA with the FTA-abs and a modified TPHA [M-TPHA) ${ }^{1}$ to see how many RPR positive cases, previously regarded as false positive when confirmed by TPHA alone, became positive when tested by M-TPHA and FTA-abs methods.

\section{Methods}

Regardless of their titres, we selectively chose 206 TPHA negative, 50 borderline positive, and 24 definite positive specimens from the positive RPR sera. Of the 206 negative cases, 125 were frankly negative and 81 were negative at $1 / 80$ and positive at $1 / 40$ dilutions. All samples were retested by M-TPHA and FTAabs. The manufacturer's instructions were strictly adhered to except in TPHA, for which we chose a titre of $1 / 40$ instead of $1 / 80$ as the lowest reactive dilution.

The reagents were obtained from the following sources:

$R P R$ BBL Microbiology System, Cockeysville, Maryland, USA.

$T P H A$ Fujirebio Inc., Shinjuku-ku, Tokyo, Japan.

$M-T P H A$ Fujirebio Inc. Shinjuku-ku, Tokyo, Japan.

$F T A$-abs Zeus Scientific Inc., Raritan, New Jersey, USA.

\section{Results}

Of the 125 specimens frankly negative by TPHA, M-TPHA showed a negative result in 78 , a borderline positive in 17 , and a positive in

Results of 280 RPR positive sera tested by TPHA, M-TPHA, and FTA-abs

\begin{tabular}{|c|c|c|c|c|c|}
\hline & \multicolumn{5}{|c|}{ Dilution titre of $T P H A$} \\
\hline & $\begin{array}{l}\text { Negative } \\
n=125\end{array}$ & $\begin{array}{l}40 \\
n=81\end{array}$ & $\begin{array}{l}40-80 \\
n=50\end{array}$ & $\begin{array}{l}80 \\
n=13\end{array}$ & $\begin{array}{l}>80 \\
n=11\end{array}$ \\
\hline $\begin{array}{l}M-T P H A \\
\text { Negative } \\
40-80 \\
80 \\
>80\end{array}$ & $\begin{array}{r}78 \\
17 \\
22 \\
8\end{array}$ & $\begin{array}{l}11 \\
14 \\
33 \\
23\end{array}$ & $\begin{array}{r}1 \\
0 \\
17 \\
32\end{array}$ & $\begin{array}{r}0 \\
0 \\
1 \\
12\end{array}$ & $\begin{array}{r}0 \\
0 \\
0 \\
11\end{array}$ \\
\hline $\begin{array}{l}\text { FT } A \text {-abs } \\
\text { Negative } \\
\text { Weak positive } \\
\text { Positive }\end{array}$ & $\begin{array}{r}69 \\
4 \\
52\end{array}$ & $\begin{array}{r}1 \\
0 \\
80\end{array}$ & $\begin{array}{r}1 \\
0 \\
49\end{array}$ & $\begin{array}{r}0 \\
0 \\
13\end{array}$ & $\begin{array}{r}0 \\
0 \\
11\end{array}$ \\
\hline
\end{tabular}


30 , and the FTA-abs gave negative result in 69 , a weak positive in 4 , and a positive in 52 (table).

Of those 81 specimens that were TPHA negative at $1 / 80$ and positive at $1 / 40$ dilutions, 11 were negative, 14 borderline positive, and 56 positive using the M-TPHA; and one was negative and 80 positive using the FTA-abs. Fifty borderline positive TPHA specimens gave negative results by $\mathrm{M}$-TPHA $(n=1)$ and FTA-abs $(n=49)$. All 24 specimens positive by TPHA were positive by both M-TPHA and FTA-abs.

\section{Discussion}

Several new serodiagnostic tests for syphilis based on enzyme linked immunosorbent $(\text { ELISA) })^{2}$ and radioimmunoassay (RIA) ${ }^{4}$ have been introduced recently, but practical experience with them to date is limited. Of those well established treponemal serological tests, the FTA-abs is the most sensitive for all stages of syphilis. The TPHA is more economical and technically less complicated but is less sensitive for detecting early primary syphilis. ${ }^{56}$

A daily problem in our laboratory is the reporting of equivocal results. The aim of this investigation resolved this issue by comparing the results of the TPHA, M-TPHA, and FTAabs among the RPR positive sera with an emphasis on the negative and borderline positive TPHA cases. We found that $47\left(38^{\circ} \mathrm{o}\right)$ and $52\left(42^{\circ}{ }_{0}\right)$ of all 125 cases negative by TPHA became positive when tested by $M$ TPHA and FTA-abs, respectively. If those 81 sera positive at $1 / 40$ while negative at $1 / 80$ are also included, the figures would rise to 117 $\left(57^{\circ}\right)$ and $136\left(66^{\circ}{ }_{0}\right)$ respectively. This indicates that over half of positive RPR and negative TPHA samples would have been regarded as biologically false positive in cases in which TPHA only was used for confirmation.

We did notice a discrepancy in the results between TPHA and FTA-abs. According to most textbooks, the data shown by the two tests are comparable in all categories of syphilis except in the primary and early secondary stages. As stated by Bradford et al, ${ }^{7}$ agreement between the two tests on 4100 Venereal Disease Research Laboratory Test (VDRL) reactive sera ranged from 92 to $98^{\circ} \%$, depending on the lots of TPHA and FTA-abs used. Average agreement was $95 \%$, with $4 \%$ reactive in the FTA-abs test and $1 \%$ in the TPHA test alone.

The question is why the positivity of the TPHA in this laboratory is so much lower than that of FTA-abs. One explanation is that there are many cases of early syphilis among our tested sera. The other possibility is probably partly due to the sampling because we purposely selected the negative and borderline positive TPHA samples. We also considered whether the quality of the Fujirebio TPHA kit had been changed during the study period. We therefore decided to compare the TPHA test results in 44 cases with two other commercially available haemagglutination kits-Wellcome and Cambridge Biomedical. Their results were all, except one, in agreement with each other in terms of positivity but sometimes differed in titres. There was only one serum sample which was negative by Wellcome and positive by Cambridge Biomedical and Fujirebio.

Although, clearly, the M-TPHA has a higher sensitivity than the standard TPHA, there were still as many as 78 positive RPR sera which did not show reactivity when tested by the M-TPHA. This put us off the idea of replacing the cardiolipin test with M-TPHA for syphilis screening. The idea came from the suggestion by some authors ${ }^{89}$ who made a case for discarding cardiolipin tests and relying on M-TPHA for syphilis screening of blood donors. According to Puckett, ${ }^{10}$ in over four years their centre did not find a single cardiolipin positive and TPHA negative case of syphilis. The big difference between our and their results may be at least partly explained by the fact that they confined their samples to blood donors alone.

We conclude that as a confirmatory test the TPHA should be used not instead of but in addition to the FTA-abs. We also suggest that a titre of $1 / 40$ should be applied as the lowest reactive dilution in place of $1 / 80$, as recommended by the manufacturer's instructions (Fujirebio).

1 Barbara JAJ, Salker R, Lalji F, Davies TD, Harris JB. An economical, simplified haemagglutination test for mass syphilis screening. J Clin Pathol 1980;33:1216-8.

2 Verldkam J, Visser AM. Application of the enzyme-linked immunosorbent assay (ELISA) in the serodiagnosis of immunosorbent assay (ELISA) in the sero

3 Pope V, Hunter EH, Feeley JC. Evaluation of the microenzyme linked immunosorbent assay with Treponema pallidum antigen. J Clin Microbiol 1982;15:630-4.

4 Baughn RE, Musher DM. Radioimmunoassays for the detection of antibodies to treponemal polypeptide antigens in serum. J Clin Microbiol 1985;21:922-9.

5 Shore RN. Hemagglutination tests and related advances in serodiagnosis of syphilis. Arch Dermatol 1974;109:854-7.

6 Borobio MV. Current problems in syphilis 109:854-7. Serodiagnosis and Immunotherapy 1987;1:393-400.

7 Bradford LL, Larsen SA. Serologic tests for syphilis. In: Lennette EH, Balows A, Hausler Jr WJ, Shadomy HJ. Manual of clinical microbiology. 4th ed. Washington, DC Manual of clinical microbiology. 4th ed. Washington,

American Society for Microbiology, 1985:910-20.
8 Lesinski J, Krach J, Kadziewicz E. Specificity, sensitivity and diagnostic value of the TPHA test. Br J Vener Dis 1974;50:334-40

9 Barbara JAJ, Salker R, Lalji F, Mochnaty P. TPHA compared with cardiolipin tests for serological detection early primary syphilis. J Clin Pathol 1982;35:1394-5.

10 Puckett A, Pratt G. Syphilis screening in the Blood Transfusion Service: a report of four years' experience with the Treponema pallidum haemagglutination assay and the Treponema pallidum haemagglutination assay and the Pathol 1987;40:1337-9. 